\title{
Evaluation of the anterior meniscal displacement with reduction by High-Resolution Ultrasonography pulsed-wave Doppler mode (PWD). A pilot study
}

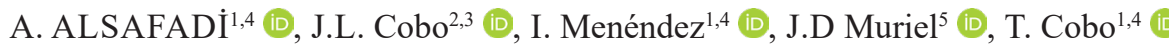

\begin{abstract}
Objective: The temporomandibular joint disorder (TMD) refers to mandibular dysfunction caused by reduced function of the primary structures that compose the temporomandibular joint (TMJ) or other structures related to the TMJ or by the interaction of these structures with each other. Ultrasound imaging (USI) can demonstrate not only soft tissue alterations but also visualize hard tissue alterations. Pulsed-wave Doppler mode is a widely used technique in the study of movable structures, particularly the vascular system.

Material and Methods: 16 patients (32 TMJ) were included in this study, grouped in 10 women's and 6 men's). An extraoral and intraoral examination of the TMJ was realized to detect anterior disc displacement with reduction. Ultrasonography highresolution examination was carried out by LOGIQ e Healthcare ${ }^{\circledR}$ with a $16 \mathrm{MHz}$ high-frequency transducer. A musculoskeletal L8$18 \mathrm{i}$ wideband linear array was used for pulsed-wave doppler mode examination.

Results: Fourteen patients presented anterior disc displacement with reduction, and 2 patients presented normal disc position. When meniscal displacement is detected, PWD mode presents a distinct waveform on the timeline of the exploration.

Conclusion: Pulsed-wave Doppler mode provides an advantage in USI, helping to confirm the existence of anterior disc displacement or crepitation. USI is a noninvasive technique with no radiation exposure. It permits static and dynamic exploration of the TMJ with a short examination time, low cost, and no contraindications or special patient preparation.

Keywords: Temporomandibular joint, Ultrasonography, Articular disc, Disc displacement, pulsed wave, Doppler.
\end{abstract}

Alaa Alsafadi (西)

alaa.alsafadi@hotmail.com

A. Alsafadi ${ }^{1,2}$, J.L. Cobo ${ }^{2,3}$, I. Menéndez ${ }^{1,4}$, J.D Muriel ${ }^{5}$, T. Cobo ${ }^{1,4}$

${ }^{1}$ Department of Surgery and Medical-Surgical Specialties, University of Oviedo, Spain

${ }^{2}$ Oral and Maxillofacial Surgery Service, Central University Hospital of Asturias, Oviedo, Spain

${ }^{3}$ Department of Biology and cellular Morphology, University of Oviedo, Spain

${ }^{4}$ Instituto Asturiano de Odontología, Oviedo, Spain

${ }^{5}$ Image center, Instituto Asturiano de Odontología. Oviedo, Spain

Submitted / Gönderilme: 22.07.2019 Accepted / Kabul: 28.06.2020

\section{Introduction}

The human temporomandibular joint (TMJ) is distinct from other synovial joints in that it is formed by two separate mesenchymal tissues(1). The TMJ consists of the mandible condyle and glenoid fossa of the temporal bone. The articular disc consists of intermediate fibrocartilage fixed to the articular capsule and the lateral margins of the condyle. However, the disc divides the articular joint cavity into two compartments: upper and lower(2). A synovial membrane covers the inner surface of the capsule and disc, except for the articular surface(3). The TMJ has a crucial role in mastication and speech, making it an essential anatomical structure of interest for dentists, orthodontists, clinicians, and radiologists(4). The term "temporomandibular joint disorder" (TMD) refers to mandibular dysfunction caused by reduced function of the primary structures that constitute the TMJ or other structures related to TMJ or by the interaction of these structures with each other(5). Pringle (6) was the first to report and define TMD in 1918. TMD affects not only the TMJ but also the masticatory system and other structures of the stomatognathic system. Overall, $41 \%$ of people exhibit at least one symptom related to TMD, and $56 \%$ have at least one related sign, which indicates that $40-60 \%$ of the general population is affected by $\operatorname{TMD}(5)$. Disc displacement is the most common intraarticular cause of TMD and results from instability between the condyle, temporal bone, and articular disc components(7). TMJ disc displacements are frequently symptomatic. However, in some individuals, disc displacement could be present with no symptoms. TMD affects women more than men, which could be referred to as the hormonal theory, which indicates that female hormones play a significant role in the pathogenesis of $\operatorname{TMDs}(8-10)$. Several studies have discussed the use of various imaging techniques as 
diagnostic methods for $\operatorname{TMD}(11,12)$. Magnetic resonance imaging (MRI) is considered the gold standard modality due to its efficacy in evaluating the soft and hard tissues of the TMJ(7). Computerized tomography (CT) enables the evaluation of the bony structures, but it is not helpful in the evaluation of soft tissue structures like the articular disc. Furthermore, CT radiation exposure limits its use as a screening method because of the large doses of ionising radiation (13).

The high demand for a non-invasive technique and allows real-time evaluation with no radiation exposure and low cost, lead a group of scientists to propose the Ultrasonography as an alternative imaging technique in 1990(7). In ultrasonography, high-frequency wave sounds (emitted by the transducer through the piezoelectric crystals) are transmitted into the human body through the transducer, the interfered echoes of the body tissues are detected and displayed on the screen(7). The main advantage of USI is the ability to perform static and dynamic studies; this feature allows the practitioner to detect the disc position more accurately than with a single static investigation. USI can demonstrate not only soft tissue alterations but also visualize hard tissue alterations(8). However, USI is not only used to detect disc position but also used for detection of joint effusion (an increase of articular space width) and condylar irregularities.

Moreover, USI has a significant advantage that has been introduced in the last generation of USI equipment, namely, the pulsed-wave Doppler (PWD) mode. This mode is widely used in the study of movable structures, particularly the vascular system. The equipment combines real-time B-mode USI with the pulsed Doppler technique, permitting the user to evaluate the differences in frequency and thereby calculate blood flow velocity in the selected vessel (14). Some studies of the TMJ have used Doppler sound analysis techniques and, recently, two-dimensional Doppler velocimetry techniques. The aim of this study was to evaluate the efficacy of PWD mode ultrasonography for the detection of ADDwr.

\section{Materials and methods}

This study evaluated 32 TMJs of 16 patients (10 women and 6 men) with age ranged between 12 to 48 years old.
These patients came to our clinic between March 2019 and June 2019 with pain in the TMJ area who were suspected of presenting TMD symptoms; the patients were otherwise in good health. The exclusion criteria of this study included previous trauma to the head and face area, prior surgery on the TMJ, orthodontic treatment, and facial deformities. Ethical approval was obtained from the ethics committee of the Instituto Asturiano de Odontologia (IAO) (Ref.IAO16-060), and this study met the criteria of Helsinki 1975, 2008 revision and Spanish legalization. Extraoral and intraoral exploration and clinical assessments of the TMJ were conducted by the same practitioner (orthodontist) per the research diagnostic criteria for temporomandibular disorders (CDC/TMD) (15). The examination included evaluating the patient's history, assessing the presence of joint sound and pain, palpating the intraoral and extraoral masticatory muscles, and measuring the mouth opening range. A clinical examination was carried out for all patients, followed by the USI B-Mode examination. Ultrasonographic examinations were carried out with a LOGIQ e ultrasound apparatus (BT12-GE Healthcare $\mathrm{ASA}$ ) with a $16 \mathrm{MHz}$ highfrequency transducer. The transducer used in this study for musculoskeletal imaging purposes was an L8-18i wideband high-frequency linear array, which has a $6.7-18.0 \mathrm{MHz}$ imaging frequency. PWD mode was applied with a frequency of $10.0 \mathrm{MHz}$, a sample volume (SV) of 2, Figure 1, a pulse repetition frequency (PRF) of $2.6 \mathrm{~Hz}$, and a wave frequency (WF) of $78 \mathrm{~Hz}$. The patient was imaged under appropriate lighting in a dental chair while in a supine position. The probe was positioned over the TMJ area perpendicular to the zygomatic arch in the transverse and longitudinal planes and tilted until the best visualization was achieved(16-19), figure 2. The images were captured in both the closed and maximally open positions. On the ultrasound images, the condylar surface and articular eminence appeared as hyperechogenic lines, while the articular disc was identified by a thin hypoechogenic band between the two lines. The relationship between the articular disc and the condyle was assessed. The disc position was classified as normal when the anterior border of the disc was located superior to the condyle and as anteriorly displaced when the anterior border of the disc was anterior to the condyle. Subsequently, a dynamic examination of the TMJ was realized using PWD mode, which allows the simultaneous display of USI and Doppler signals. 


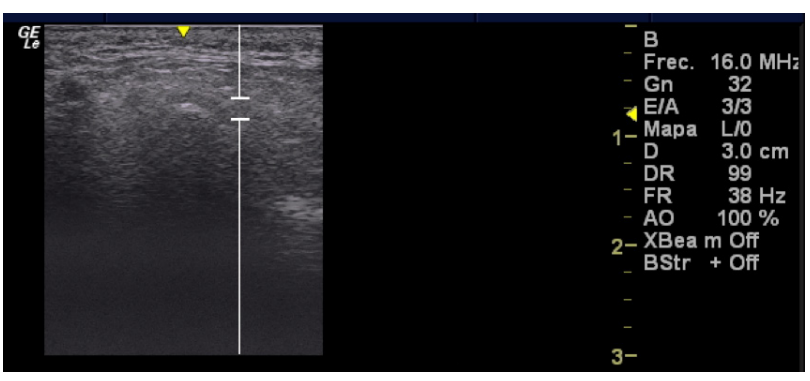

Figure 1. Sample volume of the mandibular condyle in PWD mode and analysis of condylar movement.

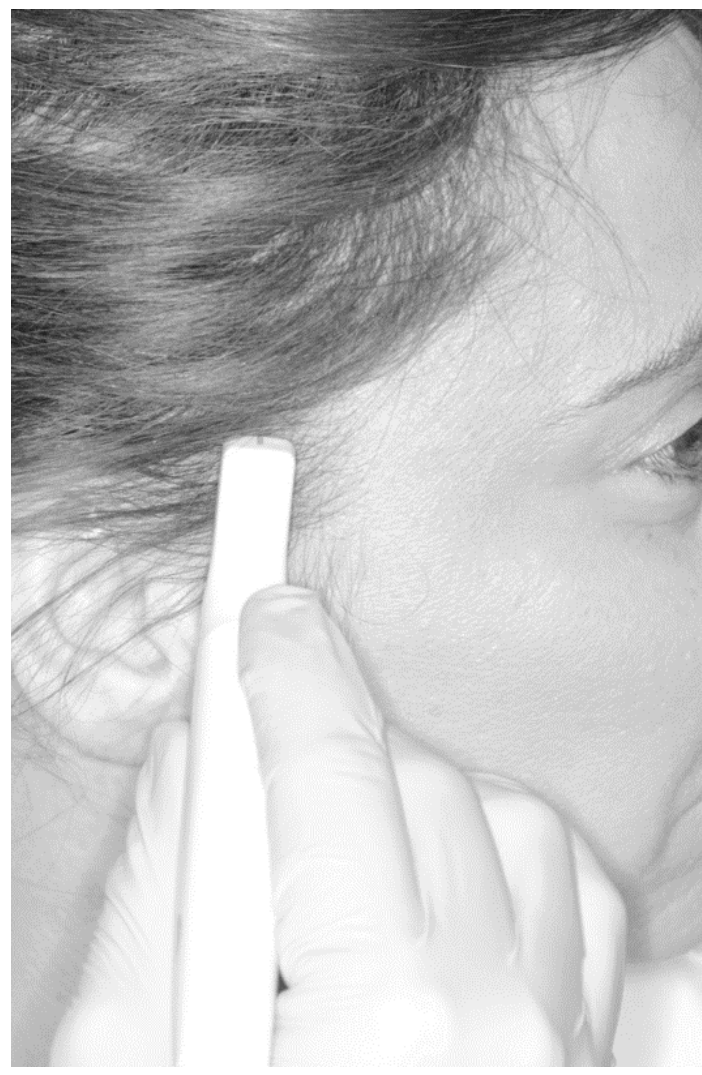

Figure 2. Patient and probe positioning in ultrasonography examination.

\section{Results}

A total of 14 patients presented ADDwr through the extraoral exploration of the TMJ. Ultrasound examination with PWD mode, confirm that fourteen patients had ADDwr, while two patients showed normal disc position.

PWD-mode USI confirmed the suspected presence of ADDwr by showing an elongated waveform on the timeline when the meniscal click occurred. In a normal patient included in this study, no alteration in the doppler spectrum was observed. Figure 3 shows a normal disc position diagnosed by PWD mode, where the examination timeline does not demonstrate any wave irregularities. While in a patient with dysfunction of the condylar-disc complex, irregular and elongated peaks were observed on examination timeline due to incorrect movement of the meniscus and condyle. However, Figures 4 from a different patient show an elongated waveform that indicates anterior disc displacement with reduction. However, Figure 5 presents a slight click of a girl with 12 years old. This click is presented on the timeline with a short and multiple waveforms. In the present work, crepitation was not detected through this study due to sample patients.

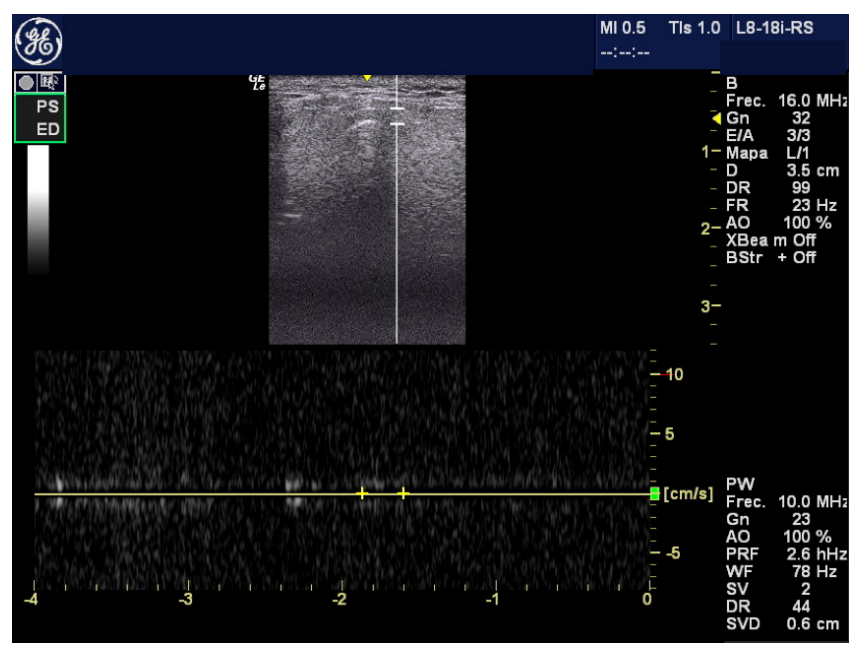

Figure 3. The patient did not present any wave irregularities on the timeline, which indicates that there was no disc displacement.

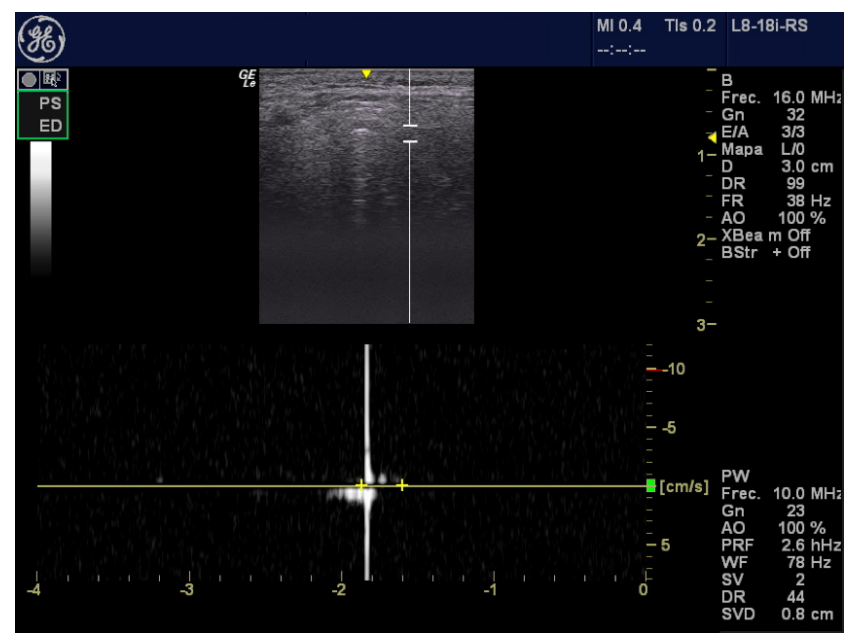

Figure 4. The patient presented anterior disc displacement with reduction. The PWD timeline shows an elongated wave at the time of the click. 


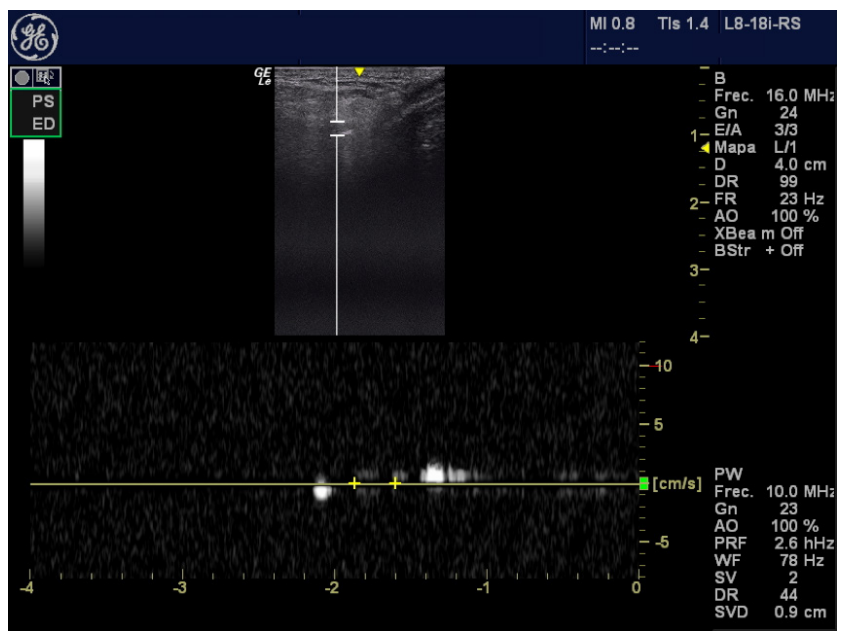

Figure 5. A 12-year-old girl presented a slight click when opening and closing her mouth. The first short wave on the left indicates the click during mouth opening, while the second short wave on the right indicate the click during mouth closing.

\section{Discussion}

The TMJ consists of the mandibular condyles, the mandibular fossa, and the articular disc. TMD is a common orofacial disorder that affects approximately $8-12 \%$ of the population, and a significant proportion of these patients seek treatment (20). TMD is divided into two groups: ID and musculoskeletal problems. ID is the most frequent type of TMD and is characterized by anterior disc displacement, joint effusion, condylar erosion, and osteoarthritis. The management of TMD starts with a clinical examination, which must be supported by the appropriate imaging modality to confirm the presence or absence of the suspected disorder, allowing the physician to make the correct treatment decision. In clinical practice, ordinary CT scans or cone-beam CT (CBCT) can help in the confirmatory diagnosis of hard tissue, while MRI can do the same for soft tissue. Many pathologies related to the head and neck, including structures such as the salivary glands, lymphatics, vascular structures, and nerves, are accessible to USI. However, reports have discussed the limitations of USI in the detection of TMD in clinical practice $(20,21)$. On the other hand, several reports confirm that USI is a useful imaging modality for the diagnosis of TMD. A study by Hayashi et al. (22) with more than 23 patients and a $10 \mathrm{MHz}$ transducer reported a Se of $63 \%$, Sp of $100 \%$ and accuracy of $72 \%$ for detecting disc displacement, while Manfredini et al. (23) reported a Se and Sp of 57\% and 74\% for detecting disc displacement, respectively. Kaya et al. (24) stated that there were no significant differences between MRI and USI in visualizing ADD, ADDwr, ADDwor, and effusion, and the findings of the two methods agreed with each other in all assessments $(p>0.05)$. Dong et al. (25) reported that patients who were suspected of having ADDwr were associated with an $89 \%$ probability of having a positive ADDwr result and a $21 \%$ chance of having a negative result after being evaluated by USI.

In comparison, the corresponding probabilities for ADDwor were $82 \%$ and $16 \%$, respectively (25). Other authors reported that ultrasound is not an effective method to distinguish between anterior disc displacement with and without reduction $(24,26)$. However, the new generation of ultrasonography equipment has a Doppler mode incorporated into it, permitting simultaneous B-mode and Doppler examination. In the dynamic exploration of the TMJ, PWD mode permits the examiner to hear and to visualize on the timeline any acoustic alteration that will produce an elongated waveform (with a distinct peak and trough), as in cases when a click caused by ADDwr occurs during the examination. This ability gives PWD an advantage in the detection of ADDwr or crepitation. It is essential to investigate sounds from the TMJ to understand TMJ dysfunction better. USI has advantages that make it superior to other imaging modalities, such as non-invasive techniques, a lack of radiation exposure, low cost, short examination time, and easily repeatable methods. Few studies have evaluated TMJ pathology through PWD mode. The recent paper about using PWD mode to study TMJ was in 2012 by A. Stagnitti et, al(14). However, the correct outcome of the examination seems to depend on operator experience as it is possible to generate many artifacts which are sometimes linked to minor methodological variations especially in the positioning and inclination of the transducer. Therefore, this study aimed to evaluate the efficacy of PWD-mode ultrasonography for the detection of ADDwr.

PWD mode uses the Doppler effect, i.e., moving objects change the characteristics of sound waves. By sending short, quick pulses of sound, it is possible to accurately measure the velocity of blood in a precise location and in real-time. In all cases, the Doppler ultrasound examination has shown the ability to differentiate between normal and pathological patients and has permitted the most significant aspects of dysfunctional to be identified (14). One must bear in mind, of course, that the examination outcome depends on operator experience, as it is possible to produce many 
artifacts, especially in the positioning and inclination of the transducer (14). A study by Stagnitti et al. (14) mentioned that the PWD examination proved particularly useful in planning and follow-up because it allowed quantification of the kinetic moment of the pathological movement, including temporal and spatial relationships. Through this study, we found that PWD mode is a useful mode that helps the physician confirm the presence of ADDwr and/ or crepitation of the mandibular articulation. This study has a small sample size; therefore, future studies of PWD mode must be conducted in large samples to confirm the effectiveness of this mode in detecting ADDwr. This work was realized because few studies were realized overusing pulsed wave mode as a diagnostic method for the detection of ADDwr. We suggest a prospective study with a more extensive patient's sample to confirm the usefulness of the PWD mode as a diagnostic method for the detection of TMD pathologies especially disc displacements and crepitation, which could allow ultrasound to take the place of MRI as the initial imaging modality for the diagnosis of TMD.

\section{Conclusion}

Ultrasonography is a useful diagnostic method for the detection of TMD. PWD mode gives ultrasound an advantage in confirming the existence of anterior disc displacement or crepitation. USI is a non-invasive technique with no radiation exposure and permits both static and dynamic exploration of the TMJ; this technique also has the advantages of short examination time, low cost, and no contraindications or special patient preparation required. A prospective study with a large patient sample is necessary to confirm the effectiveness of USI with PWD mode in diagnosing anterior disc displacement with reduction.

\section{References}

1. Bender ME, Lipin RB, Goudy SL. Development of the Pediatric Temporomandibular Joint. Oral Maxillofac Surg Clin North Am. 2018 Feb;30(1):1-9.

2. Stocum DL, Roberts WE. Part I: Development and Physiology of the Temporomandibular Joint. Curr Osteoporos Rep. 2018 Aug 9;16(4):360-8.

3. Molinari F, Manicone PF, Raffaelli L, Raffaelli R, Pirronti T, Bonomo L. Temporomandibular Joint Soft-Tissue Pathology, I: Disc Abnormalities. Semin Ultrasound, CT MRI . 2007 Jun;28(3):192-204.
4. Alomar X, Medrano J, Cabratosa J, Clavero JA, Lorente M, Serra I, et al. Anatomy of the Temporomandibular Joint. Semin Ultrasound, CT MRI. 2007 Jun;28(3):170-83.

5. Azlağ Pekince K, Çăglayan F, Pekince A. The efficacy and limitations of USI for diagnosing TMJ internal derangements. Oral Radiol. 2020 Feb 4;36(1):32-9.

6. Pringle JH. Displacement of the mandibular meniscus and its treatment. Br J Surg . 1918;6(23):385-9.

7. Y1lmaz D, Kamburoğlu K. Comparison of the effectiveness of high resolution ultrasound with MRi in patients with temporomandibular joint disorders. Dentomaxillofacial Radiol. 2019;48(5):20180349.

8. Klatkiewicz T, Gawriołek K, Pobudek Radzikowska M, Czajka-Jakubowska A. Ultrasonography in the Diagnosis of Temporomandibular Disorders: A Meta-Analysis. Med Sci Monit. 2018;24:812-7.

9. Emshoff R, Jank S, Rudisch A, Walch C, Bodner G. Error patterns and observer variations in the high-resolution ultrasonography imaging evaluation of the disk position of the temporomandibular joint. Oral Surg Oral Med Oral Pathol Oral Radiol Endod. 2002 Mar;93(3):369-75.

10. Emshoff R, Brandlmaier I, Bodner G, Rudisch A. Condylar erosion and disc displacement: Detection with highresolution ultrasonography. J Oral Maxillofac Surg. 2003 Aug;61(8):877-81.

11. Ferreira LA, Grossmann E, Januzzi E, de Paula MVQ, Carvalho ACP. Diagnosis of temporomandibular joint disorders: indication of imaging exams. Braz J Otorhinolaryngol. 2016 May;82(3):341-52.

12. Orhan K. Introduction to TMJ Imaging. In: Imaging of the Temporomandibular Joint. Springer International Publishing; 2019. p. 1-8.

13. Power SP, Moloney F, Twomey M, James K, O'Connor OJ, Maher MM. Computed tomography and patient risk: Facts, perceptions and uncertainties. World $\mathrm{J}$ Radiol. 2016;8(12):902.

14. Stagnitti A, Marini A, Impara L, Drudi FM, Lo mele L, Lillo Odoardi G. Duplex Doppler ultrasound study of the temporomandibular joint. J Ultrasound. 2012;15(2):111-4.

15. Osiewicz MA, Lobbezoo F, Loster BW, Loster JE, Manfredini D. Frequency of temporomandibular disorders diagnoses based on RDC/TMD in a Polish patient population. Cranio J Craniomandib Pract. 2018;36(5):304-10.

16. Emshoff R, Jank S, Bertram S, Rudisch A, Bodner G. Disk Displacement of the Temporomandibular Joint Sonography Versus MR Imaging American Journal of Roentgenology Vol. 2002;(June 2000):1557-62.

17. Siva Kalyan U, Moturi K, Padma Rayalu K. The Role of Ultrasound in Diagnosis of Temporomandibular Joint Disc Displacement: A Case-Control Study. J Maxillofac Oral Surg . 2018 Sep 30;17(3):383-8.

18. Thomas AE, Kurup S, Kumar SP, Chandy ML, Jose R. Diagnostic efficiency of high-resolution ultrasonography in patients with chronic temporomandibular disorders. Oral Radiol . 2016 Sep 15;32(3):160-6.

19. Su N, van Wijk AJ, Visscher CM, Lobbezoo F, van der Heijden GJMG. Diagnostic value of ultrasonography for the 
detection of disc displacements in the temporomandibular joint: a systematic review and meta-analysis. Clin Oral Investig . 2018 Sep 17;22(7):2599-614.

20. Elias FM, Birman EG, Matsuda CK, de Souza Oliveira IR, Jorge WA. Ultrasonographic findings in normal temporomandibular joints. Braz Oral Res . 2006 Jan;20(1):25-32.

21. Jank S, Zangerl A, Kloss FR, Laimer K, Missmann M, Schroeder D, et al. High resolution ultrasound investigation of the temporomandibular joint in patients with chronic polyarthritis. Int J Oral Maxillofac Surg . 2011 Jan;40(1):459.

22. Hayashi T, Ito J, Koyama JI, Yamada K. The accuracy of sonography for evaluation of internal derangement of the temporomandibular joint in asymptomatic elementary school children: Comparison with MR and CT. Am J Neuroradiol . 2001 Apr;22(4):728-34.
23. Manfredini D, Tognini F, Melchiorre D, Zampa V, Bosco M. Ultrasound assessment of increased capsular width as a predictor of temporomandibular joint effusion. Dentomaxillofac Radiol. 2003 Nov;32(6):359-64.

24. Kaya K, Dulgeroglu D, Unsal-Delialioglu S, Babadag M, Tacal T, Barlak A, et al. Diagnostic value of ultrasonography in the evaluation of the temporomandibular joint anterior disc displacement. J Craniomaxillofac Surg. 2010 Jul;38(5):3915.

25. Dong XY, He S, Zhu L, Dong TY, Pan SS, Tang LJ, et al. The diagnostic value of high-resolution ultrasonography for the detection of anterior disc displacement of the temporomandibular joint: A meta-analysis employing the HSROC statistical model. Int J Oral Maxillofac Surg. 2015 Jul;44(7):852-8.

26. Tognini F, Manfredini D, Melchiorre D, Bosco M. Comparison of ultrasonography and magnetic resonance imaging in the evaluation of temporomandibular joint disc displacement. J Oral Rehabil. 2005 Apr;32(4):248-53. 